\title{
Bone Tissue Engineering Drug Delivery
}

\author{
Pedro F. Costa ${ }^{1}$
}

Published online: 11 April 2015

(C) Springer International Publishing AG 2015

\begin{abstract}
Tissue engineering shows the potential to address the growing demand for bone tissue substitutes resulting from trauma, disease, and ageing. The utilization of drugs in combination with tissue engineering strategies has resulted in a new generation of osteoinductive scaffolds which are able to improve the speed and quality of new bone formation. This review briefly describes some of the most recent and innovative advances resulting from the combination of drug delivery methodologies with tissue engineering strategies directed at the regeneration of bone tissue. It describes some of the most commonly utilized drugs as well as some of the strategies most commonly employed to accurately direct the delivery of drugs to specific sites and cells. This review also describes several strategies which enable controlled drug release in response to specific stimuli which can be provided by the surrounding environment or medically induced.
\end{abstract}

Keywords Drug delivery · Tissue engineering · Bone

\section{Introduction}

Apart from disease and trauma, several other aspects such as population ageing, increased incidence of obesity, and sedentary lifestyles are contributing to an ever growing need for

This article is part of the Topical Collection on Molecular Biology of Skeletal Tissue Engineering

Pedro F. Costa

pedro.costa@tum.de

1 Institute for Medical Microbiology, Immunology and Hygiene, Technical University of Munich, Trogerstr. 30, 81675 Munich, Germany effective bone replacement and regeneration solutions. Tissue engineering strategies have been suggested for several decades as an alternative to commonly used but highly scarce bone grafts, given a predicted $100 \%$ increase in demand by the year 2020 [1]. Despite significant advances, tissueengineered scaffolds for bone applications have so far been relatively limited in function. A third generation of scaffolds is therefore currently envisioned, which is able to not only provide physical support for bone formation but as well actively promote the formation of large and complex bone constructs by means of incorporated chemical or biochemical agents such as drugs which can be delivered in a precise and controlled manner to the target site and to the cells involved in bone regeneration [2]. In this review, the term drug delivery will be used in a general sense, comprising as well simple drug release which is a more passive and less directed form of drug delivery. This review intends to describe some of the most recent (mainly within the last 3 years) and most innovative advances in drug delivery for bone tissue engineering applications.

\section{Commonly Employed Drugs in Bone Tissue Engineering}

As a starting point in this review, it becomes pertinent to briefly mention and describe the drugs which are most commonly utilized in bone tissue engineering. There are many molecules which play important roles in the regeneration of bone; however, some should be highlighted. The most utilized drug in bone tissue engineering studies has been by far bone morphogenetic protein (BMP). In particular, BMP-2 is known to play an essential role in bone healing, namely by influencing osteogenesis as well as vascularization [3]. This effect has been clearly demonstrated in various studies where BMP-2 
was loaded into various types of implantable carriers [4, 5•, 6 , 7]. In particular, work developed by Kolambkar et al. [8,9] has extensively demonstrated the powerful ability of BMP-2 to promote the regeneration of large bone defects by employing nanofibrous constructs combined with a modified form of alginate gel loaded with the drug. Furthermore, it has also been recently reported in a study by Ongaro et al. [10] that the action of BMP-2 onto human mesenchymal stem cells can be further enhanced through stimulation with pulsed electromagnetic fields. Another similar drug with high potential for bone regeneration is BMP-7 which has shown a strong ability to promote the repair of large load-bearing bone defects even when applied in low dosages $[11,12]$. Despite the obvious benefic effects of BMP in bone regeneration, and given the high cost and instability of BMP molecules during fabrication procedures, researchers have been alternatively resorting to more stable and less expensive synthetic drugs such as dexamethasone which is a glucocorticoid compound. In a study by Tavakoli-Darestani et al. [13], dexamethasone incorporated into hydroxyapatite scaffolds has been shown to enhance bone regeneration in rat calvarial defects when compared to nonloaded scaffolds. Another equally attractive synthetic drug currently utilized in bone tissue engineering which is able to mimic the effect of BMP-2 is simvastatin which has been shown to enhance bone formation in particular by promoting the differentiation of cells into the osteogenic lineage. This has been reported in a study by Pullisaar et al. [14] where osteogenic differentiation was achieved in human adipose tissuederived mesenchymal stem cells due to the presence of simvastatin.

Another widely utilized natural molecule in bone tissue engineering is insulin-like growth factor (IGF). IGF is well known for promoting osteogenic cell differentiation and being involved in the regulation of several key cellular processes such as proliferation, movement, and inhibition of apoptosis $[2,15,16]$. IGF-1 in particular, which is a single-chain polypeptide consisting of 70 amino acid residues, is thought to be critical in attracting osteoblast precursors to the endosteal surfaces [17]. In a study by Kim et al. [18], it was also shown that enhanced early osteoblastic differentiation can be achieved by combining the effect of IGF-1 and BMP-2 in a sequential manner. A protein superfamily involved as well in the recruitment of mesenchymal cells is transforming growth factor- $\beta$ (TGF- $\beta$ ) which also stimulates the proliferation and differentiation of those cells into osteoblasts as well as the production of extracellular matrix [19]. In a recent study by Klar et al. [20], TGF- $\beta 3$ has been shown to clearly promote the formation of bone within scaffolds implanted into intramuscular pouches in adult $P$. ursinus primates. According to the results of this same study, TGF- $\beta 3$ is also believed to promote bone formation by being involved in the upregulation of endogenous BMP-2.
Vascularization is a key component in bone regeneration since it is responsible for supplying the bone tissue with nutrients, growth factors, hormones, cytokines, and chemokines. Furthermore, vascular networks enable the removal of cellular waste products as well as communication between the bone and neighboring tissues [21]. Vascular endothelial growth factor (VEGF) is by excellence the family of molecules responsible for vascularization. The various molecules in this family are responsible for the formation of specific types of vascular structures; however, VEGF-A in particular is the main responsible for the regulation of most blood vessel growth in developing and mature individuals [22]. VEGF has been widely employed in bone regeneration strategies while loaded into various kinds of carriers ranging from 3D polymeric [23] and composite scaffolds [24] to surface coatings on titanium implants [25]. For optimal bone regeneration outcomes, VEGF can be commonly employed in combination with other drugs $[26,27]$ as well as with physiological stimuli such as compressive loading [28].

A more recent trend in drug-based bone tissue engineering is the utilization of platelet-rich plasma (PRP). Although not being exactly considered a drug, PRP can instead be considered a concentrate of platelets and various growth factors which can be easily isolated from peripheral blood by means of successive centrifugation procedures [29]. Apart from providing a suitable gel-like physical support and drug delivery vehicle [30], PRP is also intrinsically composed of growth factors such as TGF- $\beta$ as well as platelet-derived growth factor (PDGF) which is a crucial promoter of bone healing involved in the initiation of callus formation and in angiogenesis. Other factors such as VEGF have also been shown to be released from activated platelets of which PRP is composed [31]. Strategies involving the utilization of cells as a means of producing and delivering drugs in situ have been lately employed successfully in bone tissue engineering by resorting to gene therapy and co-culture strategies; however, this topic is outside the scope of this review.

Finally, apart from bone regeneration, it is also important to highlight other distinct functions of drugs in bone tissue engineering. An important example of such functions is the prevention of infections which are still a major problem encountered when open surgeries are performed. In order to solve such problem, implantable materials are currently being complemented with antibiotics. A commonly utilized antibiotic is vancomycin, an anti-gram positive bacteria drug which can be integrated into implantable materials in various forms. As examples of its versatility, vancomycin has been loaded into gel beads intended to treat osteomyelitis in rabbit tibiae [32] as well as to regenerate femoral defects in a goat model [33], it has been loaded into bone cements resulting in no significant loss of mechanical properties [34], into cancellous bone grafts for the treatment of spondylodiscitis [35] as well as into 3D scaffolds in combination with BMP-2 [36]. All of 
these strategies led to efficient elimination of infection during bone regeneration.

\section{Drug Delivery Strategies}

Several methodologies can be adopted in order to enable the release of drugs in a controlled manner from scaffolds (or materials) to their target sites (Fig. 1). The one which can be considered the most simple and straightforward consists of impregnating scaffolds with drugs (Fig. 1a). This procedure can be performed by simple immersion of a scaffold possessing adequate adsorptive properties into a drug solution resulting in the formation of a drug coating over the surfaces of the scaffold or material. This strategy was successfully employed by DeConde et al. [4] in the regeneration of marginal mandibular defects in a rat model by impregnating biomimetic scaffolds with BMP-2 prior to implantation. Another method commonly used for generating drug-delivering scaffolds consists of integrating the drug within the scaffold's material during fabrication by means of temperature-based or solvent-based processes. A simple yet effective temperature-based methodology was described in a study performed by Puga et al. [37] which consisted of melting blends of polymers and ciprofloxacin (a drug utilized in the treatment of chronic osteomyelitis) in order to generate scaffolds with sustained release of the entrapped drug. As for the solventbased approach, similar sustained drug release was achieved in a study by Kamath et al. [38] where a solvent casting and leaching technique enabled the entrapment of albumin nanoparticles loaded with resveratrol within a polymeric scaffold (Fig. 1b). In both these cases, the drug release profiles become highly dependent on parameters such as the scaffold's architecture/porosity and material's degradation rate. More advanced methodologies such as supercritical fluid technologies have also more recently been employed in the integration of drugs within materials through the combination of pressure and temperature parameters, resulting in more efficient drug loading and distribution while avoiding the use of toxic solvents or extreme temperature and $\mathrm{pH}$ processing conditions [39].

Given that bone tissue possesses unique biomechanical properties which are quite difficult to mimic artificially, many bone tissue engineering approaches (particularly for loadbearing sites) resort to the combination of two or more materials which play different roles in the generated construct (Fig. 1e). In such cases, one material may, i.e., be responsible for providing the required mechanical properties while the other material delivers drugs and/or bioactive agents which promote the regeneration process in its vicinity. Such strategy was adopted in a work by Wang et al. where mechanically strengthened $\beta$ tricalcium phosphate scaffolds were combined with a gel composed of platelet-rich plasma responsible for delivering bone healing signals [40]. In cases where the sites to be regenerated are non-load-bearing and the mechanical properties of materials and scaffolds are therefore not critical, it becomes possible to utilize materials which are more versatile in its form and functionality. Good examples of such materials are hydrogels which are mostly made of water and can be easily loaded with drugs. In a study by Kaigler et al. [41], a hydrogel was employed not only to fill generated bone defects but most importantly to promote vascularization during new bone formation. Hydrogels allow also the possibility of being applied in an injectable form becoming therefore easily placeable into inner drug target sites without the need for traumatic surgeries (Fig. 1d). In a study by Martinez-Sanchez et al. [5•], such strategy was employed for bone augmentation purposes by performing minimally invasive injection of a hyaluronic acid-based gel containing nanohydroxyapatite and BMP-2 into rat mandibles.

Another recent technology which is currently employed in the controlled release of drugs is layer-by-layer technology (Fig. 1f). It consists of the sequential formation of degradable coating layers over a given surface, which can afterwards also be decomposed to form a capsule if desired. Drugs can be entrapped in between these various layers during processing and upon implantation be released in a controlled and sequential manner as cells locally degrade the various film layers. Such technology was employed in a study by Facca et al. [6] where TGF $\beta 1$ and BMP2 were entrapped in between different layers of poly-L-lysine deposited on the surface of $1 \mu \mathrm{m}$ spherical silica particles (which were later dissolved to form a capsule) resulting in improved generation of bone tissue. Layer-by-layer methodologies can as well be applied onto conventional orthopedic implants to reduce side effects such as wear-related inflammation which is a very common complication associated with such type of implants [42]. Finally, a novel methodology which promises to revolutionize the production of drug delivery materials and devices is $2 \mathrm{D}$ and $3 \mathrm{D}$ printing (also known as additive manufacturing) (Fig. 1c). Such technologies allow to precisely print 2D and 3D patterns of drugs or drug-loaded materials enabling a more accurate control over the location and dose of drugs as well as their distance to target sites and/or progenitor cells. The effect of drugs can also nowadays be thoroughly tested within large arrays of tissue engineered constructs reproducibly biofabricated by means of additive manufacturing [43, 44]. In a work by Smith et al. [7], the importance of the distance to progenitor cells was demonstrated by printing a pattern of BMP-2-containing bioink onto the surface of circular acellular dermal matrix implants which were later implanted into mouse parietal bone defects in different positions. By having the BMP-printed implant's face positioned either away or directly facing the mouse's dura mater (source of osteoprogenitor cells), it was possible to verify that direct contact of the printed BMP-2 with the underlying dura mater 
A)

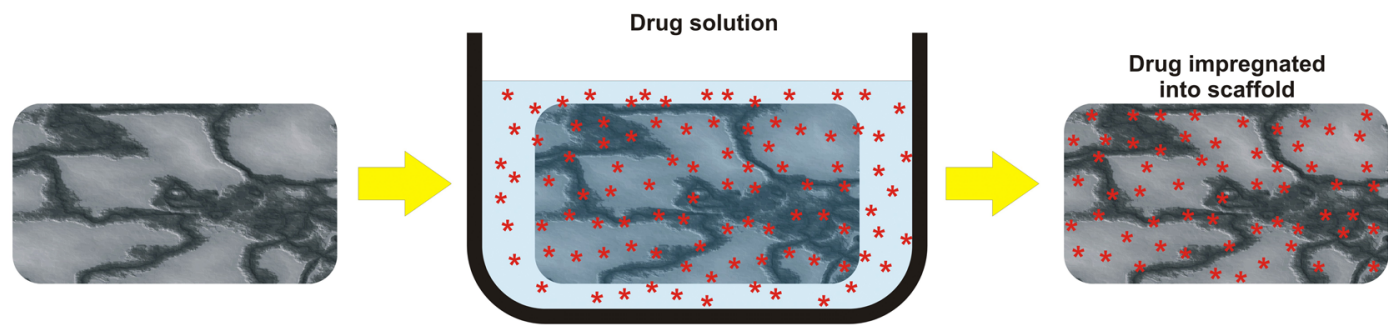

B)

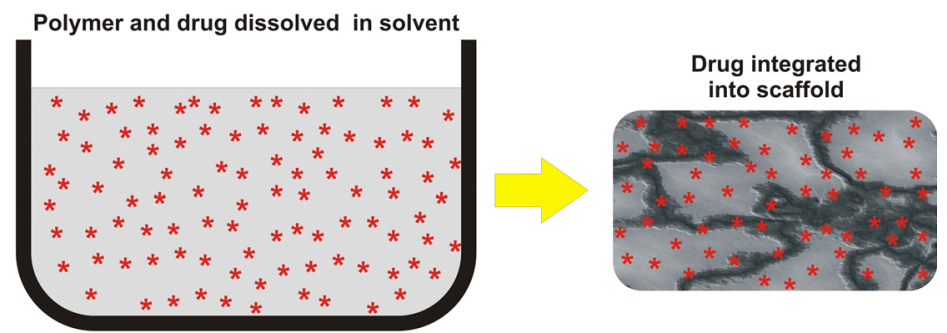

C)

D)
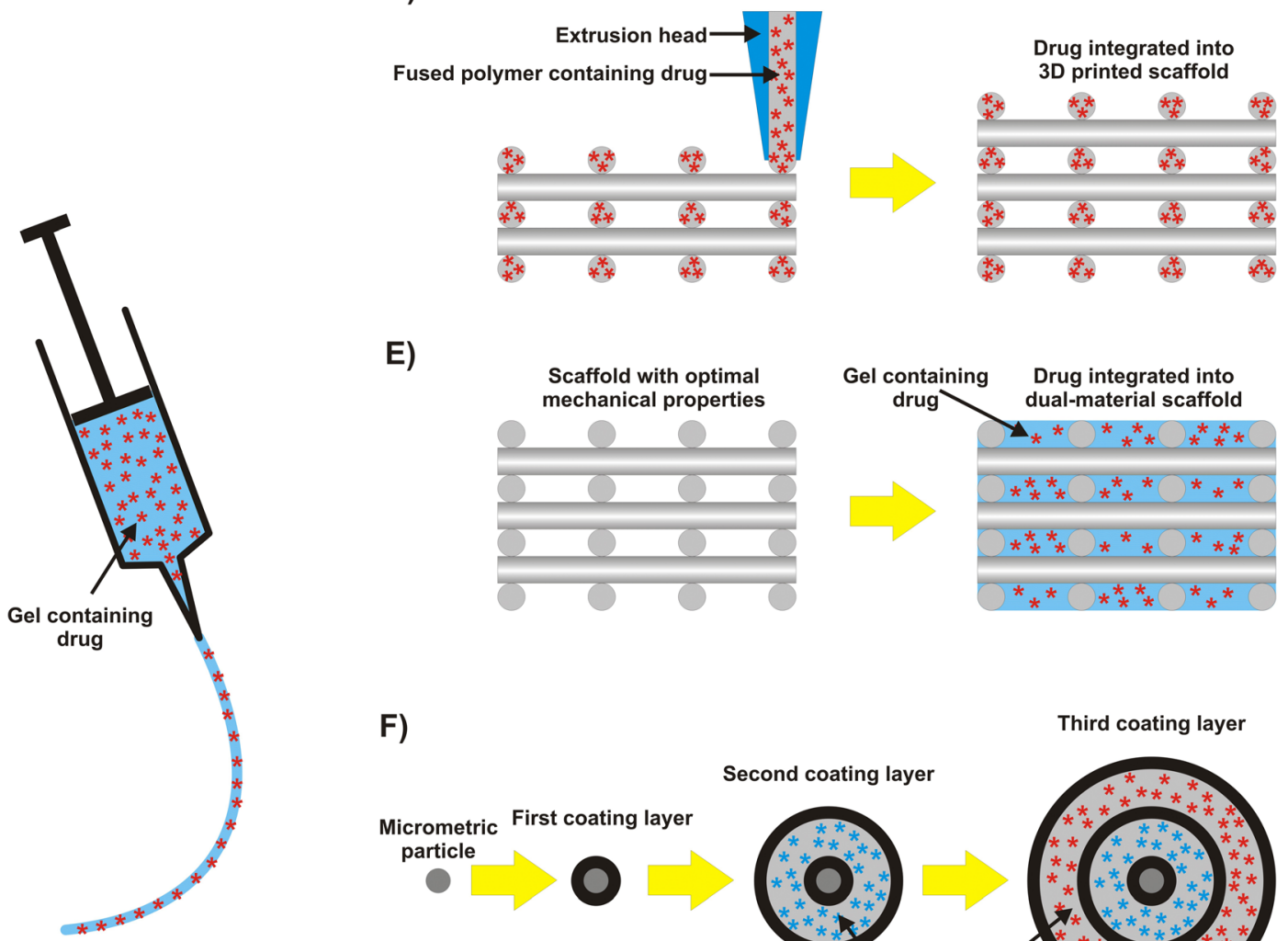

E)
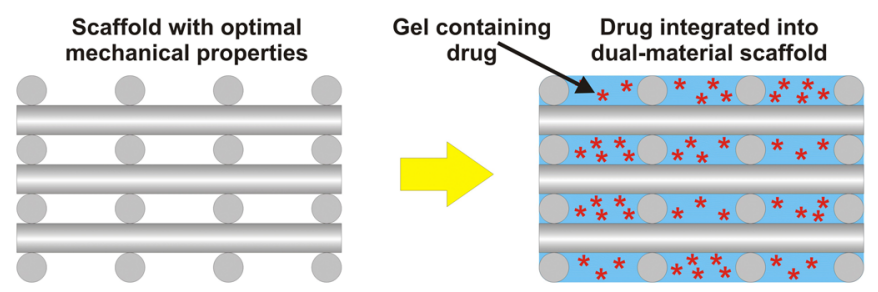

F)

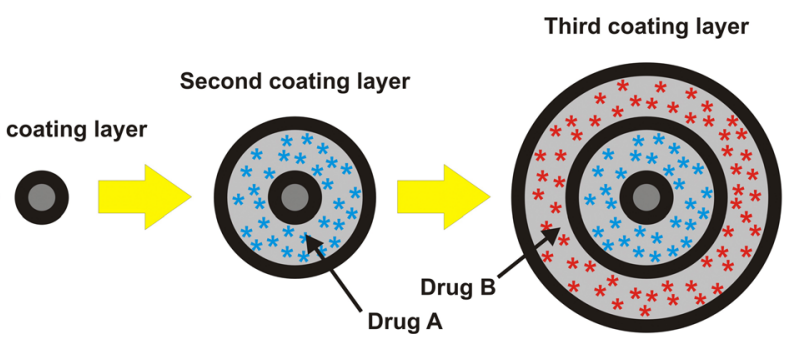

Fig. 1 Drug delivery strategies. a Impregnation of scaffold with drug, $\mathbf{b}$ drug integration during solvent-based processing of scaffold, $\mathbf{c}$ meltbased $3 \mathrm{D}$ printing of polymer containing drug, $\mathbf{d}$ injectable gel

containing drug, e combination of mechanically stable solid scaffold with drug-containing gel, $\mathbf{f}$ entrapment of two different drugs during layer-by-layer process

resulted in greater percentage of bone formation. In the particular case of 3D printing, this technology has recently become quite attractive within tissue engineering strategies due to its ability to easily generate $3 \mathrm{D}$ constructs in virtually any shape, architecture, size, and number $[45,46] .3 \mathrm{D}$ printed scaffolds have often been modified/functionalized in order to improve their osteogenic properties by means of postprocessing strategies such as, i.e., surface deposition of 
bioactive coatings or by combination with drug-releasing agents/materials [47, 48]. In a recent work employing 3D printing, a 3D scaffold composed of polycaprolactone was further embedded with a porous matrix composed of chitosan, nanoclay, and $\beta$-tricalcium phosphate in order to be able to promote the formation of mineralized matrix in vitro and as well deliver an antibiotic (anthracycline) simultaneously [48]. Despite the effectiveness of such post-processing strategies, a particular interest has more recently emerged towards the development of drug-loaded materials which can be readily and easily employed in commonly available 3D printing technologies such as fused deposition modelling. In our lab, we have recently developed a novel material which is able to release dexamethasone in a controlled manner and can as well be easily converted to filament format by means of a simplified melt-based methodology [49]. Such material can therefore be readily employed in a standardized and off-the-shelf manner in tissue engineering strategies comprising fused deposition modelling-based 3D printing while eliminating the need for further laborious drug incorporation processing steps. Apart from the important osteoinductive effect provided by the controlled release of dexamethasone, the utilization of such material by means of fused deposition modelling also enables to reduce the cost and time involved in the fabrication of drugreleasing scaffolds. Several other works employing various additive manufacturing techniques have so far been able to effectively deliver drugs showing therefore that strategies based on $2 \mathrm{D} / 3 \mathrm{D}$ printing possess the potential to become in the future an important means of efficiently delivering drugs to target sites and cells [50].

\section{Stimuli-Triggered Drug Delivery}

There are also ways to make implanted materials release drugs in a controlled manner in response to specific stimuli provided by the surrounding environment or upon medical induction. The release of drugs on demand enables more effective medical treatments given that the required concentration of each specific molecule at the site to be regenerated varies along the regeneration process. One way of enabling on demand drug release is through the utilization of materials which release drugs in response to alterations in $\mathrm{pH}$ [51]. When implanted, such materials release drugs into the surrounding environment when the surrounding physiological $\mathrm{pH}$ reaches a certain level. In a study by Vazhayal et al. [52], a combination of two non-steroidal anti-inflammatory drugs, namely ibuprofen and aspirin, was able to be released in a controlled manner from mesochanneled aerogel microspheres in response to specific $\mathrm{pH}$ changes in the surrounding environment. Responsiveness to changes in $\mathrm{pH}$ can as well be combined with changes in temperature in order to better regulate the release of drugs. In a study by Peng et al. [53], nanogels possessing $\mathrm{pH}$ and thermal dual responsiveness were developed in order to release cisplatin, an anti-cancer drug, at a rate which was slower than the one resulting from individual stimuli and therefore enabling the release of the drug for a longer period of time.

Temperature-responsive drug-releasing carriers can also be employed in strategies where ultrasound is the main determining stimulus. In a study by Staruch et al. [54•], a magnetic resonance imaging-controlled focused ultrasound system was employed in a first step to finely define a specific bone location (using the magnetic resonance imaging component) and in a second step to cause localized hyperthermia (using the focused ultrasound component) into that same exact location. By performing localized hyperthermia, this methodology, coined image-guided localized drug delivery, was able to induce the release of doxorubicin from lyso-thermosensitive liposomes previously injected into the systemic circulation of rabbits. With ultrasound being a noninvasive technology, this methodology provides an optimal way for extracorporeally triggering the release of drugs to specific sites within the patient's body.

Magnetic particles integrated into the composition of scaffolds are also currently being studied as a means to generate localized hyperthermia [55]. This is achieved by exposure to alternating magnetic fields which cause oscillation of the particle's magnetic moment and consequently result in heat release. So far, the exposure of magnetic particles to magnetic fields has shown to be highly effective in the destruction of cancer cells by means of generated hyperthermia combined with the resulting heat-triggered release of anti-cancer drugs loaded into the same particles [56]. Magnetic particles, in particular nanoparticles, show high potential in applications such as hyperthermia treatment, targeted drug delivery as well as in magnetic resonance-based imaging; however, given their novelty in this field, it becomes equally important to clearly identify any potential toxicity effects before extensive use [57].

Although not very commonly employed in bone tissue engineering strategies, light is another possible trigger for the controlled release of drugs which is worth mentioning. Light-based strategies have been extensively studied particularly in cancer treatment applications. Various light sources such as near infrared, visible or ultraviolet radiation have been employed in triggering the release of drugs either through direct induction of conformational modifications in the particles [58] or through/combined with the induction of heat [59]. Light-based strategies are however highly limited by the ability of radiation to penetrate tissues, in particular bone tissue. A future solution for this limitation could be the utilization of lasers based on two-photon technologies which can achieve longer wavelengths and therefore greater tissue penetration.

Finally, a totally new and innovative form of smart drug delivery was recently tested by Farra et al. $\left[60^{\bullet}\right]$ in a clinical trial which consisted of implanting a wirelessly controlled 
microchip which was capable of releasing drug doses on demand. Extra-corporeally generated signals were wirelessly transmitted to the implanted microchip which then released discrete drug doses once a day for 3 weeks. The drug released from the device was human parathyroid hormone (1-34), an anti-osteoporosis drug, which in this way was able to promote bone growth and thereby efficiently reversed the effect of osteoporosis felt by the postmenopausal women subjected to the trial. Such devices will most likely evolve to permanently implantable devices in the near future given the latest advances in the development of biomaterials-based electronics [61].

\section{Conclusions}

Drug delivery for tissue engineering-based bone healing applications is becoming increasingly employed. Unlike previous approaches relying solely on the osteoconductivity of materials, drug delivery-based tissue engineering approaches are currently able to actively promote the formation of bone in a much more precise and directed way. This new ability is mainly due to the more recent development of novel implantable smart materials and advanced methodologies which allow to release specific drugs in a controlled manner and according to specific stimuli. Finally, the introduction of electronics into implantable devices is another recent development which promises to radically change the paradigm of drug delivery since it will allow to fully automate the delivery of drugs according to patterns which can be remotely and digitally set on the fly, independently of physical or chemical processes.

Acknowledgments The author would like to thank the TUM University Foundation for his current postdoctoral fellowship.

\section{Compliance with Ethics Guidelines}

Conflict of Interest Pedro F. Costa declares no conflict of interest.

Human and Animal Rights and Informed Consent This article does not contain any studies with human or animal subjects performed by any of the authors.

\section{References}

Papers of particular interest, published recently, have been highlighted as:

- Of importance

1. Amini AR, Laurencin CT, Nukavarapu SP. Bone tissue engineering: recent advances and challenges. Crit Rev Biomed Eng. 2012;40(5):363-408.
2. Bose S, Roy M, Bandyopadhyay A. Recent advances in bone tissue engineering scaffolds. Trends Biotechnol. 2012;30(10):546-54.

3. Fassbender $\mathrm{M}$ et al. Stimulation of bone healing by sustained bone morphogenetic protein 2 (BMP-2) delivery. Int J Mol Sci. 2014;15(5):8539-52.

4. DeConde AS et al. Bone morphogenetic protein-2-impregnated biomimetic scaffolds successfully induce bone healing in a marginal mandibular defect. Laryngoscope. 2013;123(5):1149-55.

5. Martínez-Sanz E et al. Minimally invasive mandibular bone augmentation using injectable hydrogels. J Tissue Eng Regen Med. 2012;6(S3):s15-23. Study demonstrating how hydrogels loaded with drugs can be injected into target sites eliminating the need for surgical procedures.

6. Facca $\mathrm{S}$ et al. Active multilayered capsules for in vivo bone formation. Proc Natl Acad Sci U S A. 2010;107(8):3406-11.

7. Smith DM et al. Precise control of osteogenesis for craniofacial defect repair: the role of direct osteoprogenitor contact in BMP-2based bioprinting. Ann Plast Surg. 2012;69(4):485-8.

8. Kolambkar YM et al. An alginate-based hybrid system for growth factor delivery in the functional repair of large bone defects. Biomaterials. 2011;32(1):65-74.

9. Kolambkar YM et al. Spatiotemporal delivery of bone morphogenetic protein enhances functional repair of segmental bone defects. Bone. 2011;49(3):485-92.

10. Ongaro A et al. Pulsed electromagnetic fields stimulate osteogenic differentiation in human bone marrow and adipose tissue derived mesenchymal stem cells. Bioelectromagnetics. 2014;35(6):426-36.

11. Reichert JC et al. A tissue engineering solution for segmental defect regeneration in load-bearing long bones. Sci Transl Med. 2012;4(141):141ra93.

12. Cipitria A et al. Polycaprolactone scaffold and reduced rhBMP-7 dose for the regeneration of critical-sized defects in sheep tibiae. Biomaterials. 2013;34(38):9960-8.

13. Tavakoli-darestani R, Manafi-rasi A, Kamrani-rad A. Dexamethasoneloaded hydroxyapatite enhances bone regeneration in rat calvarial defects. Mol Biol Rep. 2014;41(1):423-8.

14. Pullisaar $\mathrm{H}$ et al. Simvastatin coating of $\mathrm{TiO}(2)$ scaffold induces osteogenic differentiation of human adipose tissue-derived mesenchymal stem cells. Biochem Biophys Res Commun. 2014;447(1): 139-44.

15. Doorn $\mathrm{J}$ et al. Insulin-like growth factor-I enhances proliferation and differentiation of human mesenchymal stromal cells in vitro. Tissue Eng Part A. 2013;19(15-16):1817-28.

16. Rosen CJ. Insulin-like growth factor I and bone mineral density: experience from animal models and human observational studies. Best Pract Res Clin Endocrinol Metab. 2004;18(3):423-35.

17. Kawai M, Rosen CJ. The insulin-like growth factor system in bone: basic and clinical implications. Endocrinol Metab Clin North Am. 2012;41(2):323-33. vi.

18. Kim S et al. Sequential delivery of BMP-2 and IGF-1 using a chitosan gel with gelatin microspheres enhances early osteoblastic differentiation. Acta Biomater. 2012;8(5):1768-77.

19. Buijs JT, Stayrook KR, Guise TA. The role of TGF-beta in bone metastasis: novel therapeutic perspectives. Bonekey Rep. 2012;1: 96.

20. Klar RM et al. The induction of bone formation by the recombinant human transforming growth factor-beta3. Biomaterials. 2014;35(9): 2773-88.

21. Saran U, Gemini Piperni S, Chatterjee S. Role of angiogenesis in bone repair. Arch Biochem Biophys. 2014;561C:109-17.

22. Stewart MW. Vascular endothelial growth factor (VEGF) biochemistry and development of inhibitory drugs. Curr Drug Ther. 2012;7(2):80-9.

23. Jabbarzadeh $\mathrm{E}$ et al. VEGF-incorporated biomimetic poly(lactideco-glycolide) sintered microsphere scaffolds for bone tissue 
engineering. J Biomed Mater Res B Appl Biomater. 2012;100(8): 2187-96.

24. Farokhi M et al. Bio-hybrid silk fibroin/calcium phosphate/PLGA nanocomposite scaffold to control the delivery of vascular endothelial growth factor. Mater Sci Eng C Mater Biol Appl. 2014;35:40110.

25. Leedy MR et al. Effects of VEGF-loaded chitosan coatings. J Biomed Mater Res A. 2014;102(3):752-9.

26. Li P et al. Synergistic and sequential effects of BMP-2, bFGF and VEGF on osteogenic differentiation of rat osteoblasts. J Bone Miner Metab. 2014;32(6):627-35.

27. Farokhi $\mathrm{M}$ et al. Sustained release of platelet-derived growth factor and vascular endothelial growth factor from silk/calcium phosphate/PLGA based nanocomposite scaffold. Int J Pharm. 2013;454(1):216-25.

28. $\mathrm{Wu} \mathrm{X}$ et al. Vascular endothelial growth factor and physiological compressive loading synergistically promote bone formation of tissue-engineered bone. Tissue Eng Part A. 2013;19(21-22): 2486-94.

29. Malhotra A et al. Can platelet-rich plasma (PRP) improve bone healing? A comparison between the theory and experimental outcomes. Arch Orthop Trauma Surg. 2013;133(2):153-65.

30. Berner A et al. Biomimetic tubular nanofiber mesh and platelet rich plasma-mediated delivery of BMP-7 for large bone defect regeneration. Cell Tissue Res. 2012;347(3):603-12.

31. Battinelli EM, Markens BA, Italiano Jr JE. Release of angiogenesis regulatory proteins from platelet alpha granules: modulation of physiologic and pathologic angiogenesis. Blood. 2011;118(5): 1359-69.

32. Xing $\mathrm{J}$ et al. Anti-infection tissue engineering construct treating osteomyelitis in rabbit tibia. Tissue Eng Part A. 2013;19(1-2): 255-63.

33. Chang $\mathrm{Z}$ et al. An anti-infection tissue-engineered construct delivering vancomycin: its evaluation in a goat model of femur defect. Int J Med Sci. 2013;10(12):1761-70.

34. Tsai YF, et al. Effects of the addition of vancomycin on the physical and handling properties of calcium sulfate bone cement. Process Biochem. (0).

35. Anagnostakos K, Koch K. Pharmacokinetic properties and systemic safety of vancomycin-impregnated cancellous bone grafts in the treatment of spondylodiscitis. Biomed Res Int. 2013;2013:358217.

36. Doty HA et al. Composite chitosan and calcium sulfate scaffold for dual delivery of vancomycin and recombinant human bone morphogenetic protein-2. J Mater Sci Mater Med. 2014;25(6):1449-59.

37. Puga AM et al. Hot melt poly-epsilon-caprolactone/poloxamine implantable matrices for sustained delivery of ciprofloxacin. Acta Biomater. 2012;8(4):1507-18.

38. Kamath MS et al. Polycaprolactone scaffold engineered for sustained release of resveratrol: therapeutic enhancement in bone tissue engineering. Int $\mathrm{J}$ Nanomedicine. 2014;9:18395.

39. de Matos MB et al. Dexamethasone-loaded poly(epsiloncaprolactone)/silica nanoparticles composites prepared by supercritical $\mathrm{CO} 2$ foaming/mixing and deposition. Int J Pharm. 2013;456(2):269-81.

40. Wang $\mathrm{C}$ et al. Preparation of a new composite combining strengthened $\beta$-tricalcium phosphate with platelet-rich plasma as a potential scaffold for the repair of bone defects. Exp Ther Med. 2014;8(4): 1081-6.

41. Kaigler D, Silva EA, Mooney DJ. Guided bone regeneration using injectable vascular endothelial growth factor delivery gel. J Periodontol. 2012;84(2):230-8.
42. Keeney M et al. Mutant MCP-1 protein delivery from layer-bylayer coatings on orthopedic implants to modulate inflammatory response. Biomaterials. 2013;34(38):10287-95.

43. Costa PF. Biofabricated constructs as tissue models: a short review. J Mater Sci Mater Med. 2015;26(4):1-6.

44. Costa PF, et al. Additively manufactured device for dynamic culture of large arrays of 3D tissue engineered constructs. Adv Healthcare Mater. 2015.

45. Costa PF et al. Automating the processing steps for obtaining bone tissue-engineered substitutes: from imaging tools to bioreactors. Tissue Eng Part B Rev. 2014;20(6):567-77.

46. Costa PF et al. Biofabrication of customized bone grafts by combination of additive manufacturing and bioreactor knowhow. Biofabrication. 2014;6(3):035006.

47. Costa $\mathrm{PF}$ et al. Advanced tissue engineering scaffold design for regeneration of the complex hierarchical periodontal structure. J Clin Periodontol. 2014;41(3):283-94.

48. Chen $\mathrm{M}$ et al. Fabrication and characterization of a rapid prototyped tissue engineering scaffold with embedded multicomponent matrix for controlled drug release. Int J Nanomedicine. 2012;7:4285-97.

49. Costa PF et al. 3D printed scaffolds with controlled release of dexamethasone for bone regeneration. Tissue Eng Part A. 2014;20(Supp. 1):56-7.

50. Moulton SE, Wallace GG. 3-dimensional (3D) fabricated polymer based drug delivery systems. J Control Release. 2014.

51. Aina $V$ et al. New formulation of functionalized bioactive glasses to be used as carriers for the development of ph-stimuli responsive biomaterials for bone diseases. Langmuir. 2014;30(16):4703-15.

52. Vazhayal $L$ et al. Mesochanneled hierarchically porous aluminosiloxane aerogel microspheres as a stable support for ph-responsive controlled drug release. ACS Appl Mater Interfaces. 2014;6(17):15564-74.

53. Peng $\mathrm{J}$ et al. Controlled release of cisplatin from $\mathrm{pH}$-thermal dual responsive nanogels. Biomaterials. 2013;34(34):8726-40.

54. Staruch R, Chopra R, Hynynen K. Hyperthermia in bone generated with MR imaging-controlled focused ultrasound: control strategies and drug delivery. Radiology. 2012;263(1):117-27. Study combining magnetic resonance imaging and ultrasound to provoke hyperthermia into accurately defined inner tissue locations resulting in localized release of a drug from circulating liposomes.

55. Zhang $\mathrm{J}$ et al. 3D-printed magnetic Fe3O4/MBG/PCL composite scaffolds with multifunctionality of bone regeneration, local anticancer drug delivery and hyperthermia. J Mat Chem B. 2014;2(43): 7583-95.

56. $\mathrm{Xu} \mathrm{Y}$ et al. Multifunctional magnetic nanoparticles for synergistic enhancement of cancer treatment by combinatorial radio frequency thermolysis and drug delivery. Adv Healthcare Mater. 2012;1(4): 493-501.

57. Kim JE, Shin JY, Cho MH. Magnetic nanoparticles: an update of application for drug delivery and possible toxic effects. Arch Toxicol. 2012;86(5):685-700.

58. Tong $\mathrm{R}$ et al. Photoswitchable nanoparticles for triggered tissue penetration and drug delivery. J Am Chem Soc. 2012;134(21): $8848-55$.

59. You $\mathrm{J}$ et al. Effective photothermal chemotherapy using doxorubicin-loaded gold nanospheres that target EphB4 receptors in tumors. Cancer Res. 2012;72(18):4777-86.

60. Farra R et al. First-in-human testing of a wirelessly controlled drug delivery microchip. Sci Transl Med. 2012;4(122):122ra21. Clinical trial demonstrating the feasibility of implanting electronic devices which release drugs according to wirelessly transmitted commands.

61. Muskovich M, Bettinger CJ. Biomaterials-based electronics: polymers and interfaces for biology and medicine. Adv Healthcare Mater. 2012;1(3):248-66. 